\title{
Molecular crystal approach for $\pi$-conjugated polymers: from PPP Hamiltonian to Holstein model for polaron states
}

\author{
Stéphane Pleutin ${ }^{\dagger *}$ and Jean-Louis Fave* \\ ${ }^{\dagger}$ Max-Planck-Institut für Physik Komplexer Systeme, Nöthnitzer Straße 38, D-01187 Dresden \\ * Groupe de Physique des Solides, 2 place Jussieu, 75251 Paris cedex 05
}

(October 30, 2018)

\begin{abstract}
Starting from the $\pi$-electron Pariser-Parr-Pople (PPP) Hamiltonian which includes both strong electron-phonon and electron-electron interactions, we propose some strongly correlated wave functions of increasing quality for the ground state of conjugated polymers. These wavefunctions are built by combining different finite sets of local configurations extended at most over two nearest-neighbour monomers. With this picture, the doped case with one additional particle is expressed in terms of quasi-particle. Thus, the polaron formation problem goes back to the study of a Holstein like model.
\end{abstract}




\section{INTRODUCTION}

The nature of the first excited states of conjugated polymers is an important and still unsolved question in condensed matter sciences [1]. Knowing if they are band to band excitations or exciton states, if polarons, bipolarons or solitons are stable quasiparticles in the doping case, are fundamental issues for the understanding of the electronic properties of these compounds.

The low-lying excited states are supposed to be suitably described by the well-known $\pi$ electron Pariser-Parr-Pople (PPP) Hamiltonian. This model Hamiltonian takes into account both strong electron-phonon and electron-electron interaction terms yielding only exact numerical solutions for the smallest oligomers [2]. For the thermodynamic limit, the situation remains unclear since the calculations for the ground state and the excited states, including electron correlations, are uneasy to achieve and some drastic approximations are needed [3].

However, a first qualitative understanding of this complicated physics can be done by the use of some simplified Hamiltonian. For instance the Rice-Gartstein's molecular exciton model [ [4], similar to previous works [5], is useful for a qualitative description of the linear absorption of conjugated polymers. On the other hand, the molecular Holstein's model gives a simplified picture of the polaron states [6,7].

Recently, an approximate scheme to build the ground and the first excited states has been proposed [8]. With this method, starting from the PPP Hamiltonian, one reaches a Rice-Gartstein like model; the excitations relevant for linear absorption are then easy to obtain and the results are comparable with those from more tedious methods [9]. In this paper, we will show that the same procedure permits to derive formally, from the very same PPP Hamiltonian, the simple molecular Holstein's model for the polaron state in

conjugated polymers. Polarons are thought to be important for the understanding of the charge transport in these compounds, and the possibility to study these non-linear states at a correlated level in an easy formalism is needed and valuable.

We choose a simple dimerized linear chain as an effective model for conjugated polymers; 
this chain is characterized by $r_{d}$ and $r_{s}$, the double and single bond lengths, respectively. Extending our method to a realistic geometry is straightforward but the essential of the physics is reached with this simplified picture. Let us now briefly introduce the PariserParr-Pople Hamiltonian which is our starting point

$$
H_{P P P}=-\sum_{n, \sigma} t_{n, n+1}\left(c_{n, \sigma}^{\dagger} c_{n+1, \sigma}+c_{n+1, \sigma}^{\dagger} c_{n, \sigma}\right)+\frac{1}{2} \sum_{n, m, \sigma, \sigma^{\prime}} V_{n, m}\left(c_{n, \sigma}^{\dagger} c_{n, \sigma}-\frac{1}{2}\right)\left(c_{m, \sigma^{\prime}}^{\dagger} c_{m, \sigma^{\prime}}-\frac{1}{2}\right)
$$

where $c_{n, \sigma}^{\dagger},\left(c_{n, \sigma}\right)$ is the creation (destruction) operator of an electron in site $n$ with spin $\sigma ; t_{n, n+1}$ is the hopping term which includes via the electron-phonon interaction a linear dependence upon the length of the bond $(n, n+1)$ [3, 10]. In the case of a dimerized linear chain, this dependence gives two distinct hopping integrals $t_{d}$ and $t_{s}$ for the double and the single bonds respectively $\left(\left|t_{d}\right|>\left|t_{s}\right|\right)$; they could be written as $t_{d / s}=t_{0}\left(1 \pm \frac{\alpha}{2 t_{0}} \delta\right)$ where $t_{0}$ is the hopping integral without dimerization, $\alpha$ is the electron-phonon interaction and $\delta$ is a measure of the dimerization giving the difference of the lengths of single and double bonds [10]. The Coulomb term is parametrized following Ohno, where the effect of the $\sigma$ electrons is considered via a phenomenologic screening, $V_{n, m}=\frac{U}{\sqrt{1+0,6117 r_{n, m}^{2}}}$ where $r_{n, m}$ is the distance (in $\stackrel{A}{A}$ ) between two electrons localized on site $n$ and $m$ [11]. We also write this term as $V\left(r_{n, m}\right) \equiv V_{n, m}$ and $V=V\left(r_{d}\right)$ for convenience.

In view to link up the PPP Hamiltonian and the molecular crystal models, the RiceGardstein and Holstein models, we choose the monomer self-consistent orbitals as basis functions - this is the so-called exciton-basis [2]. This choice is of course led by the dimerization. In our case, the monomers are the double-bonds and their self-consistent orbitals are associated with the following creation (destruction) operators for the bonding and antibonding orbitals: $B_{n, \sigma}^{(\dagger)}=\frac{1}{\sqrt{2}}\left(c_{2 n, \sigma}^{(\dagger)}+c_{2 n+1, \sigma}^{(\dagger)}\right)$ and $A_{n, \sigma}^{(\dagger)}=\frac{1}{\sqrt{2}}\left(c_{2 n, \sigma}^{(\dagger)}-c_{2 n+1, \sigma}^{(\dagger)}\right)$; here $n$ indexes the double bonds.

With this specific choice of local basis operators, the electronic configurations are built by combining different kinds of local configurations (LC) [2,12]. In order to get a tractable model, we truncate the Hilbert space by choosing a small set of different LC which will 
be the elemental building blocks for the electronic configurations [12]. These LC are the so-called generative local configurations (GLC) in [8].

We may notice that this method shows some similarities with the Valence Bond method used efficiently for the studies of oligomers [13] but with the important difference that atomic sites are replaced by monomer units with internal electronic structure (double bonds here). The configurations build from GLC are diagonal with respect to the hopping term $t_{d}$ on contrary to the Valence Bond configurations which are diagonal with respect to the Coulomb term. Each GLC is a set of several Valence Bond diagrams, chosen to be the adequate ones for a reasonable description of polymer states.

In this work, we first improve the proposed ground state of ref. [8] by enlarging the set of electronic configurations used to describe it (section II). Second, we consider the case with an extra electron on the chain and show that, if one authorizes small lattice distortions around the extra particle, our treatment allows to reach quite naturally a Holstein like model but expressed in terms of many-body particle states (section III).

\section{THE GROUND STATE}

We keep as GLC for the ground state the LC which appear the most relevant in calculations performed on small oligomers [2]. In ref [8], only three LC were considered; they are named F-LC, D-LC and $\mathrm{Ct}_{1}^{-}$-LC and are schematically represented in figure (11.a). This approximation could appear rather abrupt, but it is sufficient to get a correct qualitative picture of the linear absorption spectra as it was shown in [8]; moreover, even at this level of approximation, the results are quantitatively comparable with the results of more tedious calculations [9]. In this work, we propose some natural improvements to this first approximation by extending the set of GLC.

In a first improvement, we add to the previous set of GLC, the so-called Triplet-Triplet

LC, TT-LC, shown in figure (11.b), where two nearest-neighbour (n.n.) localized triplets are combined into a singlet. Together with the three first LC, they are the major constituents of 
the ground state wave function in small cluster calculations [2]. In a second improvement, we enlarge again the set of GLC by including in it the LC which interact directly with the four previous selected ones (figure1.c).

In the following, only the first case is treated explicitely. We develop in full detail our proposed way to get the ground state wave function with the four selected GLC. The case with the complete set of LC represented in figure (1) can be treated following the same scheme; only the obtained results are then given.

First, we introduce the four GLC, their associated creation operators and their energies.

- The named F-LC is associated with the creation operator

$$
F_{n}^{\dagger}=B_{n, \uparrow}^{\dagger} B_{n, \downarrow}^{\dagger}
$$

This define the lowest LC in the range of parameter of interest; therefore we choose as reference state

$$
\left|0>=\prod_{n} F_{n}^{\dagger}\right| \text { Vacuum }>
$$

where $\mid$ Vacuum $>$ denotes the state without any $\pi$ electron. The state $\mid 0>$ is the ground state considered in the molecular crystal approaches [4,5]; there, the linear dimerized chain is simply identified to an one-dimensional crystal of ethylene without any electronic correlations.

With respect to $\mid 0>, F_{n}^{\dagger}=I_{n}^{\dagger}$ which is simply the identity operator. In the following, all the creation operators and the energies are defined with respect to $\mid 0>$.

- The named D-LC is associated with the creation operator

$$
D_{n}^{\dagger}=A_{n, \uparrow}^{\dagger} A_{n, \downarrow}^{\dagger} B_{n, \uparrow} B_{n, \downarrow}
$$

and with energy given by $\epsilon_{d}=4 t_{d}$. 
The $\mathrm{F}$ and D-LC describe the dynamics of the $\pi$-electrons coupled by pairs into each monomer: the two electrons are independent in F-LC, whereas D-LC introduces intramonomer electronic correlation. In the strong dimerization limit, these two LC are sufficient to give a good approximation of the ground state; the system is then very close to a true molecular crystal. For small or intermediate dimerization, it is however necessary to consider more extended LC or, in other words, some fluctuations around the molecular crystal limit. This is done by introducing two more LC extended over two n.n. monomers.

- The named $\mathrm{Ct}_{1}^{-}-\mathrm{LC}$ is associated with the creation operator

$$
C t_{n}^{\dagger}=\frac{1}{2}\left(A_{n+1, \uparrow}^{\dagger} B_{n, \uparrow}+A_{n+1, \downarrow}^{\dagger} B_{n, \downarrow}-A_{n, \uparrow}^{\dagger} B_{n+1, \uparrow}-A_{n, \downarrow}^{\dagger} B_{n+1, \downarrow}\right)
$$

and with energies given in the case of a linear dimerized chain by $\epsilon_{c t}=2 t_{d}+V-$ $\frac{1}{4}\left(V\left(r_{s}\right)+2 V\left(r_{s}+r_{d}\right)+V\left(2 r_{d}+r_{s}\right)\right)$. The last term, in bracket, is the attractive interaction between the electron and the hole due to the long-range part of the Ohno potential.

The $\mathrm{Ct}_{1}^{-}$-LC introduces n.n. intermonomer charge fluctuations, reproducing the conjugation phenomenon in a minimal way.

- Last, the named TT-LC is associated with the creation operator

$$
\begin{aligned}
T T_{n}^{\dagger}= & \frac{1}{\sqrt{3}}\left(A_{n, \uparrow}^{\dagger} B_{n, \downarrow} A_{n+1, \downarrow}^{\dagger} B_{n+1, \uparrow}+A_{n, \downarrow}^{\dagger} B_{n, \uparrow} A_{n+1, \uparrow}^{\dagger} B_{n+1, \downarrow}+\frac{1}{2}\left(A_{n, \uparrow}^{\dagger} B_{n, \uparrow} A_{n+1, \uparrow}^{\dagger} B_{n+1, \uparrow}+\right.\right. \\
& \left.\left.A_{n, \uparrow}^{\dagger} B_{n, \uparrow} A_{n+1, \downarrow}^{\dagger} B_{n+1, \downarrow}+A_{n, \downarrow}^{\dagger} B_{n, \downarrow} A_{n+1, \uparrow}^{\dagger} B_{n+1, \uparrow}+A_{n, \downarrow}^{\dagger} B_{n, \downarrow} A_{n+1, \downarrow}^{\dagger} B_{n+1, \downarrow}\right)\right)
\end{aligned}
$$

and with energy given by $\epsilon_{t t}=4 t_{d}-(U-V)$.

In this LC, two Triplets appearing in n.n. monomers are combined into a singlet (figure(11.b)). It was shown to be important for the first time in the work of Schulten and Karplus 14 where it was recognized as a major constituent of the low-lying 
excitations, the famous $2 A_{g}^{-}$state, optically forbidden. In the ground state, which is our interest here, the importance of this LC can be comparable to the D-LC one [2].

We may notice that a similar treatment for the PPP Hamiltonian was proposed a few years ago to study the spin-charge separation mechanism in the limit of strong dimerization [15].

With our choice of four GLC, all possible electronic configurations are then build up. They are characterized by the number of $\mathrm{D}, \mathrm{Ct}_{1}^{-}$and TT-LC, $n_{d}, n_{c t}$ and $n_{t t}$ respectively, and by the positions of these different GLC. The positions of the $\mathrm{D}, \mathrm{Ct}_{1}^{-}$and TT-LC are labelled by the coordinates $z(k)\left(k=1, . ., n_{d}\right), y(j)\left(j=1, . ., n_{c t}\right)$ and $x(i)\left(i=1, . ., n_{t t}\right)$ respectively. The necessary non-overlapping condition between LC is supposed to be fulfilled all along the paper - the LC behave as hard core bosons. The electronic configurations are then expressed as

$$
\left|x(1), \ldots, x\left(n_{t t}\right), y(1), \ldots, y\left(n_{c t}\right), z(1), \ldots, z\left(n_{d}\right)>=\prod_{i=1}^{n_{t t}} \prod_{j=1}^{n_{c t}} \prod_{k=1}^{n_{d}} T T_{x(i)}^{\dagger} C t_{y(j)}^{\dagger} D_{z(k)}^{\dagger}\right| 0>
$$

The GLC are all neutral local configurations, therefore the energy of (7) is independent of the relative positions between LC and entirely determined by the number of each GLC.

$$
E\left(n_{t t}, n_{c t}, n_{d}\right)=n_{t t} \epsilon_{t t}+n_{c t} \epsilon_{d}+n_{d} \epsilon_{d}
$$

At this point, we have to mention an incorrect statement in [8] where it is saying that the energy of the configurations made of $\mathrm{F}, \mathrm{D}$ and $\mathrm{Ct}_{1}^{-}$-LC depends on the relative positions of the $\mathrm{Ct}_{1}^{-}$-LC. This statement is actually wrong, however, this simplification goes in favor of our treatment (indeed, it was not possible to do calculations with this statement and finally the energy (8) was also adopted in [8]).

The way we choose to diagonalize the PPP Hamiltonian in the reduced Hilbert space spanned by the electronic configurations (7) follows from 8 . First, we reorganize the configurations (7). We make linear combinations from the states with $n_{d}$ D-LC, $n_{t t}$ TT-LC localized at sites $x(1), \ldots, x\left(n_{t t}\right)$ and $n_{c t} \mathrm{Ct}_{1}^{-}$-LC localized at sites $y(1), \ldots, y\left(n_{c t}\right)$. Since we 
are interested, at the end of the day, only by the lowest state in energy (the ground state), we can consider only the linear combinations of highest symmetry

$$
\left|x(1), \ldots, x\left(n_{t t}\right), y(1), \ldots, y\left(n_{c t}\right), n_{d}>=\frac{1}{\sqrt{C_{n_{d}}^{N-2\left(n_{t t}+n_{c t}\right)}}} \sum_{\{z(k)\}} \prod_{k=1}^{n_{d}} D_{z(k)}^{\dagger} \prod_{i=1}^{n_{t t}} \prod_{j=1}^{n_{c t}} T T_{x(i)}^{\dagger} C t_{y(j)}^{\dagger}\right| 0>
$$

where the summation is carried over the $C_{n_{d}}^{N-2\left(n_{t t}+n_{c t}\right)}$ possible configurations. The energy of these combinations is still given by (8).

The states (9) interact between them by the following term

$$
\begin{gathered}
<x(1), \ldots, x\left(n_{t t}\right), y(1), \ldots, y\left(n_{c t}\right), n_{d}\left|H_{P P P}\right| x(1), \ldots, x\left(n_{t t}\right), y(1), \ldots, y\left(n_{c t}\right), n_{d}+1>= \\
\sqrt{\left(n_{d}+1\right)\left(N-2\left(n_{t t}+n_{c t}\right)-n_{d}\right)} \frac{U-V}{2}
\end{gathered}
$$

The tri-diagonal matrix, where the diagonal part is given by (8) and the off-diagonal part by $(10)$ can be divided into sub-matrices characterized by $n_{c t}$ localized $\mathrm{Ct}_{1}^{-}$-LC and $n_{t t}$ localized TT-LC but with a variable number of D-LC, $n_{d}\left(n_{d}=0, \ldots, 2\left(n_{c t}+n_{t t}\right)\right)$; these sub-matrices can be separately diagonalized and it is easy to show that the resulting lowest states are given by the following expression

$$
\begin{gathered}
\mid x(1), \ldots, x\left(n_{t t}\right), y(1), \ldots, y\left(n_{c t}\right)>^{c}=\sum_{n_{d}=0}^{N-2\left(n_{t t}+n_{c t}\right)} a^{N-2\left(n_{t t}+n_{c t}\right)-n_{d}} b^{n_{d}} \sqrt{C_{n_{d}}^{N-2\left(n_{t t}+n_{c t}\right)}} \\
\mid x(1), \ldots, x\left(n_{t t}\right), y(1), \ldots, y\left(n_{c t}\right), n_{d}>
\end{gathered}
$$

with energy expressed as

$$
E^{c}\left(n_{t t}, n_{c t}\right)=n_{t t} \epsilon_{t t}+n_{c t} \epsilon_{c t}+\left(N-2\left(n_{t t}+n_{c t}\right)\right) \epsilon_{c}
$$

where

$$
\epsilon_{c}=2 t_{d}-\frac{1}{2} \sqrt{16 t_{d}^{2}+(U-V)^{2}}
$$

The coefficients $a$ and $b$ of (11) are given by $a=\frac{U-V}{\sqrt{4 \epsilon_{c}^{2}+(U-V)^{2}}}$ and $a^{2}+b^{2}=1$. With these expressions, the double bonds free of $\mathrm{Ct}_{1}^{-}$- and TT-LC are correlated independently. The upper-script, $c$, in (11) is for correlated. $\epsilon_{c}$ is called intramonomer correlation energy. 
The next step toward the evaluation of the ground state is to retain, among all the states resulting from the previous sub-diagonalizations, only the lowest ones given by (11). This approximation is well justified since the energy difference between these states and the corresponding lowest excited ones is given by the quantity $\sqrt{16 t_{d}^{2}+(U-V)^{2}}$ which is rather high for usual parameters with a value around $10 \mathrm{eV}$. We then reorganized the states (11) into collective excitations of highest symmetry

$$
\left|n_{t t}, n_{c t}>^{c}=\left[C_{n_{t t}+n_{c t}}^{N-n_{t t}-n_{c t}} C_{n_{t t}}^{n_{t t}+n_{c t}}\right]^{-\frac{1}{2}} \sum_{\{x(i), y(j)\}}\right| x(1), \ldots, x\left(n_{t t}\right), y(1), \ldots, y\left(n_{c t}\right)>^{c}
$$

still associated with the energy (12) and where the summation runs over the $C_{n_{t t}+n_{c t}}^{N-n_{t t}-n_{c t}} C_{n_{t t}}^{n_{t t}+n_{c t}}$ possible configurations. The ground state is then expressed as a linear combination

$$
\left|G S>=\sum_{n_{t t}, n_{c t}} X_{n_{t t}, n_{c t}}\right| n_{t t}, n_{c t}>^{c}
$$

where the coefficients $X_{n_{t t}, n_{c t}}$ are determined by solving the following secular equation

$$
\begin{gathered}
I\left(n_{t t}, n_{c t}-1\right) X_{n_{t t}, n_{c t}-1}+\left(n_{t t} \epsilon_{t t}+n_{c t} \epsilon_{c t}-2\left(n_{t t}+n_{c t}\right) \epsilon_{c}-E\right) X_{n_{t t}, n_{c t}}+I\left(n_{t t}, n_{c t}+1\right) X_{n_{t t}, n_{c t}+1}+ \\
{\left[n_{t t}\left(n_{c t}+1\right)\right]^{\frac{1}{2}} n_{t t} \frac{\sqrt{3}}{2} t_{s} X_{n_{t t}-1, n_{c t}+1}+\left[n_{c t}\left(n_{t t}+1\right)\right]^{-\frac{1}{2}} n_{c t} \frac{\sqrt{3}}{2} t_{s} X_{n_{t t}+1, n_{c t}-1}=0}
\end{gathered}
$$

where

$$
I\left(n_{t t}, n_{c t}\right)=\sqrt{\left(n_{c t}+1\right) \frac{\left(N-2\left(n_{t t}+n_{c t}\right)-1\right)\left(N-2\left(n_{t t}+n_{c t}\right)\right)}{N-n_{t t}-n_{c t}}} a^{2} t_{s}
$$

The equation (16) is not solvable with the interaction term (17). Next, and last, we do an approximation on the term $I\left(n_{t t}, n_{c t}\right)$ by assuming

$$
I\left(n_{t t}, n_{c t}\right) \simeq \sqrt{\left(n_{c t}+1\right)\left(\frac{N-1}{3}-n_{t t}-n_{c t}\right)} \sqrt{3} a^{2} t_{s}
$$

This is a very good approximation of (17), if the number of GLC extended over two monomers, $n_{2}=n_{t t}+n_{c t}$, is small [8]. Consequently, this treatment will be justified if in the final wave function, the most important configurations are the ones with a small 
value of $n_{2}$; this is actually the case as it can be seen from the work of ref. [8] and as it appears, at the end of the day, in this study.

With the last simplification, the problem is mapped onto $(N-1) / 3$ independent threelevel systems. One write

$$
\begin{aligned}
& X_{n_{t t}, n_{c t}}= \sqrt{C_{n_{t t}+n_{c t}}^{\mathbf{E}(\mathbf{N}-\mathbf{1}) / \mathbf{3})} C_{n_{t t}}^{n_{t t}+n_{c t}}} y_{n_{t t}, n_{c t}} \\
& \text { with }\left\{\begin{array}{l}
y_{n_{t t}, n_{c t}} / y_{n_{t t}+1, n_{c t}}=\gamma \\
y_{n_{t t}, n_{c t}} / y_{n_{t t}, n_{c t}+1}=\zeta
\end{array}\right.
\end{aligned}
$$

where $\mathbf{E}$ takes the integer part, $\gamma$ and $\zeta$ are real constants to be determined. Inserting this definition in (16) and after some algebraic manipulations one finds that the problem goes back to calculate the lowest eigenvalue, $\epsilon$, of the following 3 by 3 matrix

$$
\left(\begin{array}{ccc}
0 & \sqrt{3} a^{2} t_{s} \\
\sqrt{3} a^{2} t_{s} & \epsilon_{c t}-2 \epsilon_{c} & \frac{\sqrt{3}}{2} t_{s} \\
\frac{\sqrt{3}}{2} t_{s} & \epsilon_{t t}-2 \epsilon_{c}
\end{array}\right)
$$

The ground state energy is then simply divided into two different components

$$
E_{G S}=N \epsilon_{c}+\frac{N-1}{3} \epsilon
$$

The first part is the intramonomer correlation energy defined by the first subdiagonalization; it is obtained by correlating independently the $N$ double bonds. The second part is the intermonomer fluctuation energy defined by the second subdiagonalization; it is obtained by considering $(N-1) / 3$ identical and independent effective three level systems defined by the matrix (20). Finally, the ground state wave function is clarified by the following two equations

$$
\gamma=\frac{a^{2} t_{s}}{\epsilon} \quad, \quad \zeta=\frac{2 a^{2}}{\sqrt{3}} \frac{\epsilon-\epsilon_{t t}}{\epsilon}
$$

The resulting wave function contains, as the energy, two different kinds of components: the first ones localize electrons by pairs in the double bonds; the second ones introduce n.n. intermonomer fluctuations, charge fluctuations by means of $\mathrm{Ct}_{1}^{-}-\mathrm{LC}$ and spin fluctuation by means of TT-LC. 
The ground state proposed above may be easily improved by adding new local configurations extended over two n.n. double bonds. For example, one can include the whole LC represented in the figure (11); the LC of (11.c) are the ones directly coupled to the others. The strategy is then the same. First, one takes care of the intramonomer correlation; second, one builds the collective excitations of highest symmetry; third, one approximates the part of the resulting interaction connecting configurations which differ by only one LC extended over two monomers in the way of (18). The problem is then equivalent to consider $(N-1) / 3$ independent seven-level systems; $\epsilon$ is then the lowest eigenvalue of the associated 7 by 7 matrix.

In order to test our assumptions from which we propose several ground state wave functions in the form of (15), we do comparisons, first, for the Su-Schrieffer-Heeger (SSH) model. For this model, similar to (14) but without the complicated Coulomb term [16], the exact result is well known [16,[17. We compare this result with successively the results given by the model ground state of ref [8], the hereafter so-called model I, the one with in addition the TT-LC, the model II, and, last, the model with all the GLC represented in figure (10), the model III. We make comparison in function of the dimerization parameter $x=\frac{\alpha}{2 t_{0}} \delta$. The results are shown in Table I where the percentage of the exact energy for our successive approximations are given. For $x=1$, the case of complete dimerization, the three models give obviously the exact result. For $x=0$, the case without dimerization, one gets around $92 \%$ of the total energy. A-priori in this limit, one would expect less accurate results since the charge fluctuations of longer range than one play a role; they contribute in fact only in the missing $6 \%$. For $x=0.15$, a value often attributed to the polyacetylene, one gets around $97 \%$ of the total energy. In conclusion, our approximation seems rather good for realistic cases, within this independent electron model.

Next, we do also comparisons for the Hubbard model which is well known to be exactly solvable in one dimension [18]; this is the model (1) with $\alpha=0$ and where only the on-site electron-electron interaction, $U$, is retained. For $U=0$, one gets the SSH model without dimerization for which we obtained around $92 \%$ of the total energy (see Table I). Starting 
from these values, the agreement monotonically decreases when $U$ increases to finally get for infinite $U$, between $77 \%$ and $79 \%$ of the total energy, depending on the model (I, II or III) under consideration. This discrepancy shows that important LC are missing especially in the strong $U$ limit; for instance, it is easy to see, just by energetic considerations, that, for large enough $U$, the TTT-LC, which is singlet made by three localized triplets, the TTTTLC, which is singlet made by four localized triplets, and so on, may become important for the ground state wave function. With our specific choice of basis set completely localized on the double bonds, the dimerization parameter, $x$, is crucial; the more it will be important, the more our treatment will be relevant to become exact for a complete dimerization. In the Hubbard model, the dimerization is simply missing. If $\alpha \neq 0$, the energy of the LC made from localized triplets increases making our approximations more and more reasonable.

Last, we do comparison for the so-called extended Peierls-Hubbard model; this is the model (11) but with only the Hubbard term, $U$, and the n.n. interaction $V$, with the assumption that $V=V\left(r_{d}\right)=V\left(r_{s}\right)$ [20]. On the contrary to the two previous models, this model is not integrable, also, we do comparisons with calculations performed with the Density-Matrix Renormalization Group (DMRG) technique [22]; a very recent review of the advances related to this method may be found in [23]. The DMRG calculations have been done by E. Jeckelmann [21] following the method developed in ref [20]. We compare our approximate results with an extrapolation of the energy per unit cell made from calculations for different lattice lengths up to two hundreds double bonds. The calculations are performed for a reasonable choice of parameters, $U=4 t_{0}$ and $V=t_{0}$. The results for several values of the dimerization parameter are confined in table II. We see that the errors are always less than $20 \%$ and are around $13-10 \%$ for realistic parameters. In our opinion, the agreements obtained here are satisfactory considering the relative simplicity of the wave functions proposed in this work. Moreover, with these approximate wave functions, some analytical insights are now possible which is very new in this range of parameters, appropriated for conjugated polymers.

We do not compare for the moment our results with calculations made for the com- 
plete PPP Hamiltonian. Nevertheless, since the remaining long range terms of the Coulomb potential are of smaller importance than the other terms of the Hamiltonian, one can reasonably expect only small quantitative changes in the results obtained with the extended Peierls-Hubbard model by using the full PPP Hamiltonian.

Before closing this section, we may say that our wave functions are not variational since, in the way we choose to diagonalize the model, we do two successive sub-diagonalizations with some approximations. However, it is possible to build some variational wave-functions very similar to (15). By the way, works are already done to propose a variational version of the model II [24] and other are in progress for the model III [25]. In other way, a very efficient Matrix-Product-Ansatz is also proposed in [26]. Compared to the work developed in [24], one can say that our proposed way to diagonalize the PPP-Hamiltonian in the selected sub-Hilbert space is a very good approximation for appropriated parameters.

\section{POLARONIC STATES}

In this part, we consider the situation with one additional charge. We treat explicitely the case of an additional electron but, the case of the removal of one electron can be treated exactly in the same way. We show that this problem can be describe, with some approximations, in terms of quasi-particles which obey to simple effective Hamiltonian. For a rigid lattice, we get a one dimensional tight binding Hamiltonian. If one authorizes some distortions of the lattice around the extra particle, we get at second order in the distortion coordinates, a Holstein like model [6]. In both cases, the parameters of these one-electron models are related to the PPP one's.

In this work, we are not attempted to derive quantitative results. Our goal, based on semi-quantitative results, is to open up a way between a true many-body model given by the PPP-Hamiltonian and more simple one-electron models as the Holstein's model for polaronic states. Because it is not possible to solve the PPP model and since the important physical ingredients for an understanding of conjugated polymers are still not fully recognized [1], 
the derivation of more effective models is needed in order to get some physical insight. This work, and the very related one of ref. [8], goes in this direction.

For convenience, we choose in this part the simplest description for the ground state given by the model I, using F, D and $\mathrm{Ct}_{1}^{-}$-LC. Since the model I already contains the most important local constituents for the ground state wave function, namely the $\mathrm{F}$ and $\mathrm{Ct}_{1}^{-}$-LC, we believe the results would not changed dramatically with a better description - by using the model II or III. Then, if we define

$$
\left|n_{d}, n_{c t}>=\left[C_{n_{c t}}^{N-n_{c t}} C_{n_{d}}^{N-2 n_{c t}}\right]^{-1 / 2} \sum_{\{y(i), z(j)\}} C t_{y(1)}^{\dagger} \cdots C t_{y\left(n_{t}\right)}^{\dagger} D_{z(1)}^{\dagger} \cdots D_{z\left(n_{d}\right)}^{\dagger}\right| 0>
$$

where the summation is over the $C_{n_{c t}}^{N-n_{c t}} C_{n_{d}}^{N-2 n_{c t}}$ possible configurations, the ground state wave function is simply written as

$$
\left|G S>=\sum_{n_{c t}=0}^{N_{c t}} a_{c t}^{N_{c t}-n_{c t}} b_{c t}^{n_{c t}} \sqrt{C_{n_{c t}}^{N_{c t}}} \sum_{n_{d}=0}^{N-2 n_{c t}} a_{c}^{N-2 n_{c t}-n_{d}} b_{c}^{n_{d}} \sqrt{C_{n_{d}}^{N-2 n_{c t}}}\right| n_{d}, n_{c t}>
$$

where $N_{c t}=\mathbf{E}\left(\frac{N-1}{3}\right), a_{c}=\frac{(U-V)}{\sqrt{4 \epsilon_{c}^{2}+(U-V)^{2}}}, a_{c}^{2}+b_{c}^{2}=1, a_{c t}=\frac{\sqrt{3} a_{d}^{2} t_{s}}{\sqrt{\epsilon_{t}^{2}+12 a_{d}^{4} t_{s}^{2}}}$ and $a_{c t}^{2}+b_{c t}^{2}=1 . \epsilon$ is then the lowest eigenvalue of the 2 by 2 matrix obtained from (20) by suppressing the effective level corresponding to the TT-LC [8]. For a typical choice of parameters relevant for conjugated polymers [10], the most probable LC is the F-LC $\left(a_{c}^{2} \simeq 0.98\right.$ and $\left.a_{c t}^{2} \simeq 0.25\right)$; typical values for the energies are given by $\epsilon_{c} \simeq-0.26 \mathrm{eV}$ and $\epsilon \simeq-1.26 \mathrm{eV}$.

An additional charge disturbs the electronic cloud more or less strongly depending on the system under consideration. It could be a local distortion where the extra particle rearranges the system in short distances to create around it what it is called polarization cloud; this is the case for usual semi-conductors. On the contrary, it could be a complete rearrangement of the system as for strongly correlated systems [19]. In our case, the first behaviour is concerned and a quasi-particle picture is reached.

We describe the perturbations caused by the extra-particle - the polarization cloud - by introducing a new set of LC more or less extended, which we call Charged Local Configurations (C-LC); the term "charged" means that they contain explicitely the extra-particle. Some example of C-LC, extended over one, two and three double bonds are shown in figure 
(2) where the extra-electron is represented by the thick arrow. In the case of a "macroscopic" rearrangement of the electronic structure - as it could be the case for strongly correlated systems - the maximum extension of the relevant C-LC would be of the order of the system size. In our case, this critical size is of the order of some monomer units only.

All around these C-LC, we assume the electronic structure unchanged with respect to the ground state; therefore, we consider such charged configurations - strictly speaking, these are linear combinations of electronic configurations but we adopt the proposed terminology for convenience -

$$
\begin{gathered}
\left|\alpha_{n}>=\right| N_{L}>\otimes\left|C_{n}^{\alpha}>\otimes\right| N_{R}> \\
\left|\beta_{n, n+1}>=\right| N_{L}>\otimes\left|C_{n, n+1}^{\beta}>\otimes\right| N_{R}-1> \\
\left|\gamma_{n, n+1, n+2}>=\right| N_{L}>\otimes\left|C_{n, n+1, n+2}^{\gamma}>\otimes\right| N_{R}-2>
\end{gathered}
$$

where, $\left|C_{n}^{\alpha}>,\right| C_{n, n+1}^{\beta}>$ and $\mid C_{n, n+1, n+2}^{\gamma}>$ are some C-LC extended over one, two and three nearest-neighbour double bonds respectively, $\mid N_{L}>\left(\left|N_{R}>,\right| N_{R}-1>, \mid N_{R}-2>\right)$ is the part on the left (right) of the C-LC, described in the same way as $\mid G S>$. With this crude description, a C-LC acts as a dramatic boundary which simply interrupts the chain: the system is separated into two chains both described exactly as the ground state; the boundary contains explicitely the extra-particle within a defined C-LC. The more extended C-LC are inserted in the ground state in the same way as (25).

With our approximation, the energy of each charged configuration (as (25)) is given by the addition of two different terms. The energy of the isolated C-LC and the energy of the external parts to the left and to the right of the C-LC. Since $\mid N_{L}>$ and $\mid N_{R}>$ are neutral, the external parts don't interact via the Coulomb potential with the extra-particle. However, the configurations (25) must be improved for more quantitative results. Indeed, in a better description, because of the presence of the P-LC, the relative weight of the F, D and $\mathrm{Ct}_{1}^{-}$-LC, controlled by the coefficients $a_{c}, b_{c}, a_{c t}$ and $b_{c t}$ should depend on their positions on the chain. Moreover, with an additional particle, the electron-hole symmetry is broken. All the LC used in $\mid N_{L}>$ and $\mid N_{R}>$ are in the same sector of symmetry - the proper one for the building of the ground state. This is the case, for instance, of the $\mathrm{Ct}_{1}^{-}-\mathrm{LC}$ where the 
charge transfer on the right and on the left are of the same importance. In the presence of the P-LC these two charge transfers are no longer equivalent; the symmetry is broken and this implies a coulombic interaction between the P-LC and the external parts. These effects, not considering in this work, would certainly modify the polarisation cloud in a sensitive way. One can say, in other words, that the 'embedding" of the C-LC, due to the part $\mid N_{L}>$ and $\mid N_{R}>$ of (25), are not treated efficiently in this work. We believe this is here the main point to be improved in the future for more quantitative results.

For usual values of the PPP model, one kind of charged configurations is smaller in energy than the other and in such way that a perturbative treatment is possible to do. These configurations are due to the $\mathrm{C}$-LC, referred as the $\mathrm{P}-\mathrm{LC}$ hereafter $(\mathrm{P}$ stands for Particle), associated with the following creation operator

$$
P_{n, \sigma}^{\dagger}=A_{n, \sigma}^{\dagger} F_{n}^{\dagger}
$$

and represented in figure (2.a). The extra-particle is immersed in the reference vacuum and gives the following charged configurations

$$
|n>=| N_{L}>\otimes\left|P_{n}>\otimes\right| N_{R}>
$$

where $n$ referred to the position of the P-LC, and with an energy given by $\mathcal{E}_{n}=\epsilon_{n}+\left(N^{r}+\right.$ $\left.N^{l}\right) \epsilon_{c}+\left(N^{r}+N^{l}-3\right) \frac{\epsilon}{3}$, with $N^{r}+N^{l}=N-1$ and $\epsilon_{n}=t_{d}+\frac{U}{2}+\frac{3 V}{2}$, the energy of the isolated P-LC. By comparing with (21), we see that there is a loss of intramonomer correlation energy and a loss of intermonomer fluctuation energy with respect to the ground state; indeed, the additional electron occupies a site in which one cannot place $\mathrm{D}$ and $\mathrm{Ct}_{1}^{-}$-LC. This loss of energy is more important for the more extended C-LC.

In the following, we consider explicitely only the charged configurations (27) since the effects of the other charged configurations can be taken into account by perturbation. With our approximation, because of the n.n. hopping integral, the P-LC can hop on the lattice with the help of the F-LC or the $\mathrm{Ct}_{1}^{-}$-LC. With the former, the P-LC can hop from site to site on the monomer lattice (see figure 3). 


$$
<n\left|H_{p p p}\right| n \pm 1>=J=a_{c}^{2} b_{c t}^{2} \frac{t_{s}}{2}
$$

In this expression, the product $a_{c}^{2} b_{c t}^{2}$ gives the probability to find a F-LC in the wave function (24); the factor $1 / 2$ in (28) comes from our choice to work with the monomer orbitals. Moreover, with our approximation, there exists also a n.n.n. hopping process with the help of the more extended GLC, the $\mathrm{Ct}_{1}^{-}$-LC (see figure 屯).

$$
<n\left|H_{p p p}\right| n \pm 2>=a_{c t}^{2} \frac{t_{s}}{4}
$$

The additional factor 2 in the denominator comes from the fact that only one term from the $\mathrm{Ct}_{1}^{-}$-LC (see equation (5)) is involved during the transfer; the coefficient $a_{c t}^{2}$ gives the probability to find a $\mathrm{Ct}_{1}^{-}$-LC in the ground state wave function (24). The n.n.n. transfer is of course less important than the n.n. one's. With the values for the parameters we use here, the values of these two hopping processes differ by one order of magnitude. Therefore, we neglect the n.n.n. effective hopping term in this work.

The extra-particle (P-LC) can be dressed by perturbation. Some effects of the other CLC appear then in renormalized energy and n.n. hopping term for the extra-particle. This dressing of the P-LC can be simply done by a second order perturbative treatment, giving, in one hand, the so-called polarization energy

$$
\epsilon_{p}=\sum_{\delta} \frac{t_{\delta}^{2}}{\mathcal{E}_{n}-\mathcal{E}_{\delta}}
$$

and, in the other hand, some corrections for the n.n. hopping integral $J$

$$
J_{e f f}=\sum_{\delta, \delta^{\prime}} t_{\delta} t_{\delta^{\prime}}\left(\frac{1}{\mathcal{E}_{n}-\mathcal{E}_{\delta}}+\frac{1}{\mathcal{E}_{n}-\mathcal{E}_{\delta^{\prime}}}\right)
$$

In these expressions $t_{\delta}$ and $t_{\delta^{\prime}}$ are some interacting terms between the P-LC and other CLC. The inequalities $\left|\frac{t_{\delta / \delta^{\prime}}}{\mathcal{E}_{n}-\mathcal{E}_{\delta / \delta^{\prime}}}\right|<<1$ are respected for the values of the parameters we use which guaranty the relevance of a perturbative treatment. After that, we have reached a quasi-particle picture, the quasi-particle being represented by the P-LC.

In principle, many C-LC give some contributions to the perturbative series (30) and (31). However, because the states (25) ignore many effects due to an inappropriate embedding, 
as we already mentioned, we believe it is not useful to carry out the full calculation. Consequently, we do here a simplified treatment for the dressing of the extra-particle which we believe contains anyhow the most important contributions to (30) and (31). This simplified treatment consists to consider the C-LC not embedded in the ground state defined by (24) but in a simplified vacuum made of only F-LC. Since, the F-LC is the very most important LC in the ground state (24), we believe this simplified treatment sufficient to capture the most important parts of the polarisation energy and the effective hopping term. Moreover, among the remaining charged configurations only a few are incorporated in the perturbative treatment; they are shown in the figure (2.b). By this last simplification we neglect all the C-LC shown in the figure (5) which take into account some long range polarisation effects; these C-LC are numerous but their total effect on (30) and (31) are small and they don't participate sensitively to the binding energy of the polaron state which is the main quantity we are looking for here. With this treatment, the corrections for the hopping term remain always negligible in the range of parameters of interest; we will therefore neglect these last corrections, $J_{\text {eff }}$.

After the dressing operation, we obtain formally a one particle like problem with two characteristic energy terms $E_{n}$, the site energy of the additional 'electron' with respect to the ground state, and $J$, the hopping term, which are functions of the PPP parameters: $E_{n}=\epsilon_{n}-\epsilon_{c}-\frac{2}{3} \epsilon+\epsilon_{p}$ and $J=\frac{t_{s}}{2}$. If we suppose a rigid lattice, the problem can obviously be diagonalized, giving a band centred at $E_{n}$, with a bandwidth of $4|J|$. In the case of the SSH Hamiltonian [16] and by neglecting $\epsilon$ and $\epsilon_{p}$, the bottom of the band is given by $E_{n}=\left|t_{d}-t_{s}\right|$, the exact result; with inclusion of these corrective terms, this energy becomes slightly overestimated. The effective mass associated with the P-LC is given by $m^{*} \simeq \frac{\hbar^{2}}{a^{2}} \frac{1}{t_{s}}$ ( $a$ is the unit cell length) which is of course higher than the effective mass of a free particle on the bottom of the conduction band. With the Coulomb term, $E_{n}$ increases and $m^{*}$ stays unchanged. In conclusion, for a rigid lattice, we have reached a simple tight binding Hamiltonian - the so-called Hückel model. Last, one may say that such approach is quite close in spirit of a recent work of J. Grafenstein et al., where an effective tight-binding 
model is derived at ab-initio level by means of an incremental method [27].

Now we allow a relaxation of the lattice. For simplicity, we choose a model displacement where the two 'atoms' of the same double bond move with the same amplitude $\frac{\left|x_{n}\right|}{2}$ but in opposite directions (cf. figure (6) ). The two parameters $E_{n}$ and $J$ depend now on the lattice coordinates via mainly the linear dependence of the two hopping terms, $t_{d}\left(x_{n}\right)=t_{d}-\alpha x_{n}$ and $t_{s}\left(x_{n}, x_{n+1}\right)=t_{s}+\alpha\left(\frac{x_{n}}{2}+\frac{x_{n+1}}{2}\right)$. On the contrary, the coulombic terms remain almost unchanged after a small displacement. The contributions due to these displacements to $E_{n}$ and $J$ are small so we make a linear expansion with respect to $\left\{x_{n}\right\}$ of these two quantities

$$
\begin{gathered}
E\left(x_{n}\right)=E_{n}-\alpha\left(a_{0} x_{n}+a_{1}\left(x_{n+1}+x_{n-1}\right)\right) \\
J\left(x_{n}\right)=J-\alpha b_{0}\left(x_{n}+x_{n+1}\right)
\end{gathered}
$$

where $a_{0}, a_{1}$ and $b_{0}$ are functions of PPP parameters and $\alpha$ is the electron-phonon interaction term [16]. The extra elastic constraint of the dimerized chain due to the lattice relaxation in presence of an additional charge is expressed as

$$
E_{e l}=\frac{1}{2} \sum_{n} K_{e q}\left[x_{n}^{2}+\left(\frac{x_{n}}{2}+\frac{x_{n-1}}{2}\right)^{2}\right]
$$

where $K_{e q}$, the spring constant, is defined relatively to the dimerized equilibrium structure.

The coefficients $C_{n}$ of the Holstein polaron wave function [6], $\left|\Psi_{p}>=\sum_{n} C_{n}\left(x_{n}\right)\right| n>$, are determined by minimization of the corresponding total energy, $E_{T}\left(\left\{x_{n}\right\}\right)$, with respect to the lattice coordinate $x_{n}$. At the second order in $x_{n}$ and taking into account that $\frac{\alpha}{K_{e q}} \sim 0.1 \AA$ in conjugated polymers [3], we obtain the characteristic equations of the molecular Holstein's model

$$
\begin{gathered}
{\left[F x_{n}-2 J-\epsilon\right] C_{n}+J C_{n+1}+J C_{n-1}=0} \\
x_{n} k=F\left|C_{n}\right|^{2}
\end{gathered}
$$

where the coefficients are expressed in function of the PPP parameters: $F=\left(a_{0}+2 a_{1}+4 b_{0}\right) \alpha$, $k=2 K_{e q}$ and $J=\frac{t_{s}}{2}$. By injecting (36) into (35) we obtain the non-linear Schrödinger 
equation which gives the coefficients of the wave function; the second equation connects in a simple manner these coefficients and the lattice deformation. The analytical solution of these two equations in the continuum limit [6], valid for the "large" polaron case, gives the well known polaronic wave function $C_{n}=\frac{\gamma}{\eta} \operatorname{sech}\left(\gamma\left(n-n_{0}\right)\right)$ with $E_{b}=\frac{F^{2}}{2 k}, \eta^{2}=\frac{E_{b}}{J}$ and $\gamma^{2}=\frac{\eta^{2}}{2}$; the polaron state is localized around $n_{0}$, an undetermined quantity because of the translational invariance of the system. The associated binding energy of the polaron state is given by $E_{p}=\frac{E_{b}^{2}}{12 J}$.

We evaluate these quantities for several choices of parameters by the following sequence of calculations. First we optimize the dimerized geometry referring to a spring constant, $\mathrm{K}$, relative to a hypothetical undimerized geometry [16]; then, we evaluate $K_{\text {eq }}$, calculating the second derivative of $E_{T}$ with respect to the dimerization coordinate at the geometrical equilibrium. Second, we solve the equations (35) and (36).

In the continuum version of the SSH Hamiltonian limit 28], analytical expressions have been given. Our results always overestimate the reported values. For example, with $t_{0}=2.5 \mathrm{eV}, \alpha=4.1 \mathrm{eV} \stackrel{\circ}{ }^{-1}$ and $K=21 \mathrm{eV}^{-2}$, we get $E_{p}=0.11 \mathrm{eV}$ in place of $0.064 \mathrm{eV}$. In the same manner, our method also overestimate the value of the dimerization. These overestimations occur naturally from our starting point which relies on a molecular description. Besides it has been shown that the SSH Hamiltonian is never equivalent to the Holstein's model for the dimerized linear chain [28], so that the approximations of our model cannot be expected to lead to a good agreement in this case. However our approximations will cope better when the Coulomb interaction is taken into account; then the energies of charge fluctuation components decrease with their extensions, due to the long range part of the potential. This fact is in favour of our approximation. Furthermore the value of $K$ used in this example is the appropriated one for the SSH Hamiltonian [1]6], but seems not to be in agreement with the experimental results obtained for small oligomers [3]. An higher value must be taken, favouring again our description.

If one adds the Ohno potential, the binding energy decreases: as example, for the same choice of parameters and $U=11.16 \mathrm{eV}$, we get $E_{p}=0.091 \mathrm{eV}$. Finally, taking the same 
parameters but with a more appropriate value for $K, K=41 e V \AA^{-2}$, we get a reasonable equilibrium geometry characterized by $r_{d}=1.33 \AA$ and $r_{s}=1.47 \AA$. Moreover we get the following values $F \simeq 9.5 \mathrm{eV} \AA^{-1}, J \simeq 1.1 \mathrm{eV}, k \simeq 78 \mathrm{eV} \AA^{-2}$ and the binding energy for the polaron decreases to $E_{p} \simeq 0.025 \mathrm{eV}$. In any case, we stay around traditionally adopted values.

Before closing this section, note that with such a low binding energy, expected for conjugated polymers, the quantum fluctuations of the lattice should be explicitely considered. However, it is for the moment totally hopeless to introduce additional bosonic variables in the full PPP Hamiltonian.

\section{CONCLUSION}

In conclusion, we have proposed a simplified treatment of the PPP Hamiltonian which is typically a diagonalization of this Hamiltonian in a restricted Hilbert space. The method adopted, using monomer orbitals, is a natural way to bridge the gap between small cluster and polymer calculations [2,8]. The ground state is composed of intermonomer nearestneighbour fluctuation components in the background of coupled electrons by pairs localized on monomers. Comparisons with DMRG results for the extended Peierls-Hubbard model show satisfactory agreements considering the simplicity of our proposed wave functions. The electronic excitations are then described as local perturbations moving in this "vacuum". For an appropriate set of parameters, this description gives rather good values for the dimerisation and for the energies of the excited states active in one photon spectroscopy [8]. In the doped case $(2 \mathrm{~N}+1$ particles $)$ studied here, following the adiabatic scheme proposed by Holstein [6], we show that our model leads naturally to a Holstein's polaron like problem.

However our description differs drastically from the Holstein's polaron image in the sense that it is able to describe the behaviour of a strong correlated $(2 \mathrm{~N}+1)$ particle state whereas the Holstein's model considers only the additional particle in interaction with a deformable medium. The obtained binding energy of the polaron is of the correct order of magnitude. 
Some improvements are suitable concerning, first, the ground state where more extended GLC must be considered in order to reproduce more accurately the delocalization proper to $\pi$-systems. In other hand, variational calculations based on the very same ideas are possible [24 26]. For the doped case, we believe the first point would be to improve the description of the vacuum in presence of the extra-particle. Even if it is difficult to test our derivation in part owing to a lack of accurate calculations including correlation effects, we think that our formulation keeps the essential behaviour of the considered physical phenomenon and believe that it could be useful for future more advanced studies in part because of its relative conceptual simplicity and of its ability to give analytical expressions. For example, the behaviour of polaron states in presence of a strong electric field [7], which corresponds to a common situation in electroluminescence studies, would be to consider taking into account the effects of the strongly correlated $\mathrm{N}$ particle system.

\section{ACKNOWLEDGMENTS}

We wish to thank E. Jeckelmann for giving us DMRG results prior to publication. S.P acknowledges support from the European Commission through the TMR network contract ERBFNRX-CT96-0079 (QUCEX). 


\section{REFERENCES}

[1] Primary photoexcitations in Conjugated Polymers: Molecular Exciton versus Semiconductor Band Model, edited by N.S. Sariciftci (World Scientific Publishing, Singapore, 1997).

[2] M. Chandross, Y. Shimoi and S. Mazumdar, Phys. Rev. B 59, 4822 (1999).

[3] D. Baeriswyl, D.K. Campbell and S. Mazumdar, in Conjugated Conducting Polymers, edited by H. Kiess (Springer-Verlag, Heidelberg, 1992), pp 7-133.

[4] M.J. Rice and Y.N. Gartstein, Phys. Rev. Lett. 73, 2504 (1994); Y.N. Gartstein, M.J. Rice and E. Conwell, Phys. Rev. B52, 1683 (1995).

[5] W.T. Simpson, J. Am. Chem. Soc. 77, 6164 (1955); H.C. Longuet-Higgins and J.N. Murrel, Proc. Roy. Phys. Soc. A68, 602 (1955); J.A. Pople and S.H. Walmsley, Trans. faraday Soc. 58, 441 (1962).

[6] T. Holstein, Ann. of Phys. 8, 325 (1959).

[7] M.N. Bussac, J. Dorignac and L. Zuppiroli, Phys. Rev. B55, 8207 (1997).

[8] S. Pleutin and J.L. Fave, J. Phys. Cond. Matt. 10, 3941 (1998).

[9] Z.G. Yu, R.T. Fu, C.Q. Wu, X. Sun and K. Nasu, Phys. Rev. B52, 4849 (1995).

[10] P. Tavan and K. Schulten, Phys. Rev. B36, 4337 (1987).

[11] K. Ohno, Theor. Chim. Acta 2, 219 (1964).

[12] S. Pleutin, Thesis, Paris 7 University, 1997.

[13] Z.G Soos and S. Ramasesha, in Valence Bond Theory and Chemical Structure, edited by D.J. Klein and N. Trinajstic, Elsevier, Amsterdam, 1990.

[14] K. Schulten and M. Karplus, Chem. Phys. Lett. 14, 305 (1972).

[15] D. Mukhopadhyay, G.W. Hayden and Z.G. Soos, Phys. Rev. B51, 9476 (1995). 
[16] W.P. Su, J.R. Schrieffer and A.J. Heeger, Phys. Rev B22, 2099 (1980).

[17] L. Salem, Molecular Orbital Theory of Conjugated Systems (Benjamin, London 1966).

[18] E.H. Lieb and F.Y. Wu, Phys. Rev. Lett. 20, 1445 (1968).

[19] P. Fulde, Electron Correlations in Molecules and Solids, 3rd edn. Springer Series in Solid-State Sciences (Springer, Berlin, 1995).

[20] E. Jeckelmann, Phys. Rev. B57, 11838 (1998).

[21] E. Jeckelmann, private communication.

[22] S.R. White, Phys. Rev. B48, 10345 (1993).

[23] Density-Matrix Renormalization Group, Lecture Notes in Physics, eds I. Peschel, X. Wang and K. Hallberg, Springer-Verlag, 1999.

[24] S. Pleutin, E. Jeckelmann, M.A. Martin-Delgado and G. Sierra, preprint condmat/9908062, submitted to Prog. Theo. Chem. Phys.

[25] S. Pleutin, in preparation.

[26] M.A. Martin-Delgado, G. Sierra, S. Pleutin and E. Jeckelmann, preprint condmat/9908066, submitted to Phys. Rev. B.

[27] J. Grafenstein, H. Stoll and P. Fulde, Phys. Rev. B55, 13588 (1997).

[28] D.K. Campbell, A.R. Bishop and K. Fesser, Phys. Rev. B26, 6862 (1982). 


\section{FIGURES}

a)

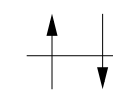

b)

c)

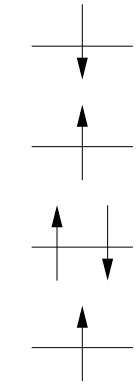

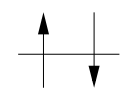
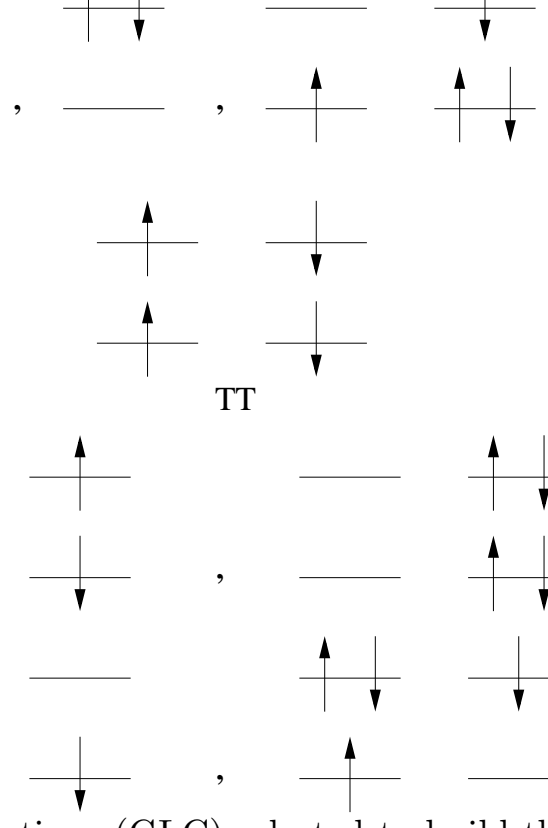

FIG. 1. Generative-Local-Configurations (GLC) selected to build the ground state wave functions. The set of GLC (a) defines the model I, the set of GLC (a+b) defines the model II and the whole set of GLC $(a+b+c)$ defines the model III.

a)
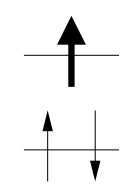

b)

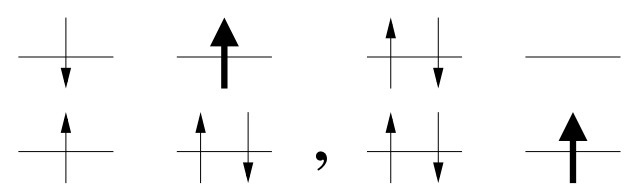

c)

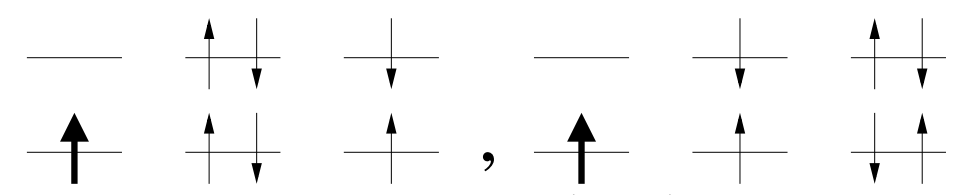

FIG. 2. Examples of Charged-Local-Configurations (C-LC) extended over one (a), two (b) and three (c) double bonds. The extra-particle is represented by the thick arrow. The quasi-particle is identified with the P-LC (a), the lowest C-LC in energy. 


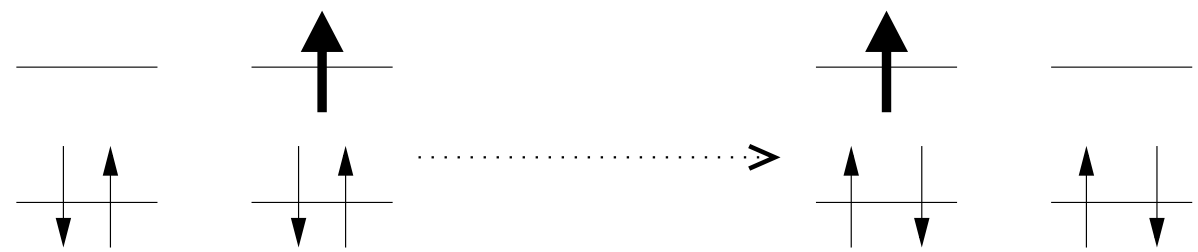

FIG. 3. Nearest-neighbour hopping process for the P-LC assisted by the F-LC

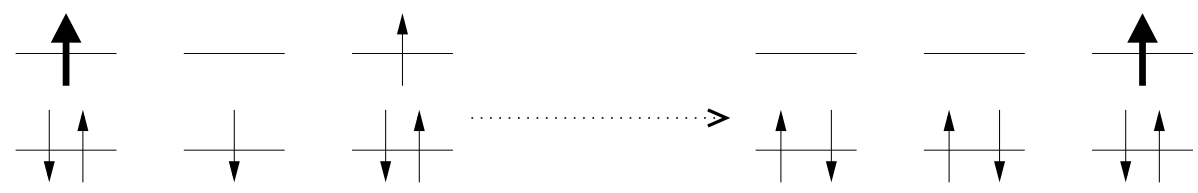

FIG. 4. Next-nearest-neighbour hopping process for the P-LC assisted by the $\mathrm{Ct}_{1}^{-}$-LC.

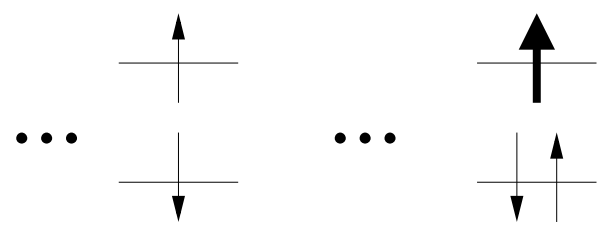

FIG. 5. Example of Local Configurations including long range polarization effects

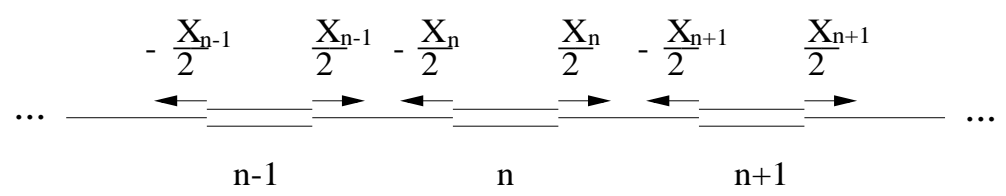

FIG. 6. Model for the lattice deformation adopted in this work. 


\section{TABLES}

TABLE I. Percentage of the exact energy obtained with the different models studied here for the S.S.H. Hamiltonian. The model I contains the F, D and $\mathrm{Ct}_{1}^{-}-\mathrm{LC}$; the TT-LC are added for the model II and the whole set of LC shown in figure (1) are considered for the model III.

\begin{tabular}{lccc}
\hline \hline & model I & model II & model III \\
$x=0$. & $91.6 \%$ & $92.1 \%$ & $92.7 \%$ \\
$x=0.15$ & $96.7 \%$ & $96.9 \%$ & $97.2 \%$ \\
\hline \hline
\end{tabular}

TABLE II. Energy per unit cell for an infinite lattice obtained with the three successive approximations (model I, II, III) and DMRG calculations for the extended Peierls-Hubbard model with $U=4 t$ and $V=t$; in the case of DMRG, the energies per unit cell are obtained from extrapolation of large cluster calculations up to 400 sites.

\begin{tabular}{lcccc}
\hline \hline & model I & model II & model III & DMRG \\
$x=0.05$ & 0.373311 & 0.369217 & 0.366820 & 0.313599 \\
$x=0.15$ & 0.306566 & 0.304317 & 0.303046 & 0.270381 \\
$x=0.25$ & 0.236707 & 0.235678 & 0.235022 & 0.213969 \\
$x=0.75$ & -0.160370 & -0.159835 & -0.159840 & -0.164925 \\
\hline \hline
\end{tabular}

